\title{
THE IMPACT OF BANNER AD STYLES ON INTERACTION AND CLICK-THROUGH RATES
}

\author{
Adam Sigel, Xavier University, siegela@xavier.edu \\ Gerald Braun, Xavier University, braun@xavier.edu \\ Mark Sena, Xavier University, sena@xavier.edu
}

\begin{abstract}
Since the introduction of the first graphical web browser on the onslaught of web advertisement, academic and corporate researchers have been interested in studying factors that impact the effectiveness of advertising methods and banner ads in particular. The click-through rate of an ad has been the standard measuring stick for assessing the effectiveness of the ad. This study examines the effect of banner style, including size and orientation, on interaction and click-through rates. Three different styles were presented on two different web sites with over ten million total impressions. For the sites and styles that we used, results seem to contradict some of the established thinking in terms of commonly accepted standards for selecting size and orientation of banner ads.
\end{abstract}

Keywords: Electronic Commerce, Banner Ads

\section{INTRODUCTION}

There has been a significant body of academic and corporate research dedicated to web advertising since graphical browsers became popular in the mid1990's, with a heavy emphasis on the study of banner ads [11]. Banner ads display promotional messages at the top, bottom, or side of a webpage with the primary purpose of attracting viewers to the advertiser's web site. While web advertising can take on many forms, including text links, sponsorships, classifieds, target sites, etc., banner advertisements have historically been the most prevalent [4, $6 \& 7]$.

Web activity, and consequently web advertising continues to grow and evolve with new technologies and increased broadband access. As of February, 2008, Nielsen/NetRatings [19] reports 221 million consumers in the US with a monthly access rate of $74 \%$. Internet advertising increased from $\$ 7.6$ billion in 2001 to $\$ 10.3$ billion in 2002 and continues to increase at a substantial rate. As the web access increases and new technologies emerge to allow for more sophisticated ads, it is important to continually analyze the effectiveness of ad campaigns. The effectiveness of banner advertisements has been traditionally measured by click-through rates - the rate at which viewers click on a banner ad to visit the target site, and there has been an increasing trend to base pricing on click-through rates [4].

Despite the allure of banner ads, their overall effectiveness has been questioned in the literature [4, 20 \& 8]. According to a study cited by Cho [4] the number of people who never look at banner ads increased from 38\% to 48\% from 1997 to 1998 and the number of people who look at banner ads often or very often decreased from $16 \%$ to $9 \%$. . Clickthrough rates have also declined significantly from $7 \%$ in 1997 to .7\% in 2002 [8]. More recently, there has been evidence that heavy Internet users tend to ignore or avoid Internet ads altogether [4].

According to Dreze and Hussherr [8], in an experiment which used an eye-tracking device, only half of their subjects remembered seeing a banner ad on a page which they viewed.

However, people who support the effectiveness of banner ads contend that the goal of most banner ads is twofold - generating direct responses (measured by click-through rates) and obtaining brand recognition through banner exposure [4]. Pharr [20] contends that "there is an amazing body of evidence....that supports the idea that Internet advertising can impact brand attitudes and feelings in the absence of clickthrough."

With so much money being spent on banner advertising, companies analyze almost every imaginable aspect of their banner campaigns. There are theories and stats on everything from creative execution to banner ad placement. Surprisingly, one area that often seems to get overlooked is also the simplest and most fundamental; the banner style (including size and orientation). The Interactive Advertising Bureau (IAB) sets guidelines for types and sizes of banner ads including full and half horizontal (leaderboard), rectangle, vertical, and skyscraper (tall vertical) [15].

This study examines banner ad size, style and orientation for three separate banner styles on two different sites. Data were collected with regard to impressions, interaction rates (roll over to launch or expand a panel), and click-through rates. 


\section{BACKGROUND}

Big brands are continuously making information technology a more significant part of their marketing strategy. Just a few short years ago, brands were seen as hip and "keeping up with technology" if they simply had a web site, no matter how rudimentary it was. Fast forward to the present, and almost every brand dedicates a sizable portion of its marketing budget to interactive marketing, spending on a wide variety of things from videos to email blasts.

Half of all marketers allot over $15 \%$ of their overall marketing budgets on campaigns, and this number is rising [22]. In fact interactive marketing spending in the U.S. is projected to more than triple over the next five years, reaching $\$ 61$ billion by 2012 -close to $20 \%$ of total ad budgets [3]. Much of this money will come at the expense of traditional media like print and TV [13].

When it comes to interactive marketing, brands spend more on banner ads than almost anything else [6, Dahener]. Brands use banners to build brand loyalty, prompt a sale, drive consumers to a website, collect personal data, offer incentives and more [22]. Companies like banner ads because they allow for measurable numbers and real-time tracking. The average click-through rate is the statistic most measured by companies when measuring banner effectiveness. The click-through rate is the percent of users who see a banner and then click on it to link to the brand's website or wherever else the advertiser wants to drive traffic. The industry average clickthrough rate is .15\% [23]. That means that, on average, for every million people that see a banner, only 1,500 click through it.

These low rates are tolerated because of the economic efficiency banner advertising provides. Brands also figure that there is some benefit just by getting impressions on a user (an impression is when a user sees the banner) even if the user may not clickthrough. That being said, brands are constantly searching for ways to increase their all important click-through rates.

There have been many studies done on ways to increase rates. For instance, research shows that incorporating video into banner ads can increase click-through rates between 2.5 and 5 times higher than building banners without video [3]. Other common suggestions to improve click-through rates include using animation, limiting the amount of text, having a strong call to action ("Click Here!" message) and using buzz words like "free" [18, 5 \& 17]. Cleland, et al. [5] found that an interactive banner using an embedded search form significantly improved click-through rate. In another study, $\mathrm{Li}$ and
Bukovac [17] found that animated banner ads resulted in better recall than still ads. In yet another study on interactive ads for children's sites which featured interactive games, results show higher clickthrough rates. Furthermore, teens, who have been shown to have relatively low rates, responded more favorably to ads featuring prizes or games [21].

Other factors that may impact click-through rates are audience and brand familiarity. Dahlen [6] found that familiar brands receive more than double the click-through rate of unfamiliar brands. However, click-through rates decreased with multiple exposures of familiar brands, but increased with multiple exposures to unfamiliar brands. In the same study, it was found that least experienced users had a higher brand awareness and brand attitude than did more experienced users.

Studies have shown that all of these elements, can have a profound impact on click-through rates. However, the banner creative is only one half of the puzzle. The other half is all about the specifics of how and where an ad is placed. And just like real estate, it's all about size and location.

The specific kinds of pages that banners run on (context) can have an even more significant effect on performance than their placement on the page. Data shows that finely targeting the placement of banner ads to run on sites where the target consumer is most likely to surf (running an ad for Madden '08 on ESPN.com) will make the ad significantly more relevant and can increase click-through rates anywhere from $30 \%$ to $300 \%$ [2 \& 18]. This targeting can even funnel down to the page level. For instance, marketing the newest Madden football game on ESPN.com might be good, but marketing it on the NFL landing page on ESPN.com might be even better.

But what if we drill down even farther, down to the most basic level? Suppose a brand has done its research and knows which websites it wants to run on. Suppose it even knows which specific pages on each website will be most effective. That's a great start, but the brand's job is not done yet. It still must also decide what size and style of banner it wants to run.

Unfortunately, there hasn't been much research done to help brands answer this question. The limited research that does exist suggests that the larger the ad, the better the click rates and purchase intent [1]. Google AdSense's Optimization team reports that the most successful ads are not only big, but wide as well. Google AdSense lists the three most effective banner sizes (in pixels) as $336 \times 280$, followed by 300x250 and then 160X 600. A study by Li and 
Bukovac [17] found larger banner ads resulted in better comprehension and a higher click-through rate than for smaller banner ads. This contradicted results from a prior study by Li [16] which found that neither ad size nor animation had an affect on clickthrough rates. Dreze and Hussherr [8] also had conflicting results with regard to ad size when they observed subject actions using eye-tracking techniques. In their initial study, they found that size could be an important factor in determining whether an ad zone is attended. However, in a follow-up study, they found that small banner ads perform just as well as large ones, and they found weak support for any affect of banner orientation (vertical or horizontal).

Most online media buys are set up to run a variety of banner sizes in specific locations. As part of a package media buy, sites usually charge different amounts based on what specific pages a banner is running on, but charge little or no differently based on the actual size or style of the banner. This is highly surprising given how finely targeted everything else is in interactive advertising and leaves a huge potential benefit for interactive advertisers. Companies could realize major benefit at no additional cost by buying the exact right size and style on each page.

\section{METHODOLOGY}

To determine whether or not a specific banner size or style is more effective than others, one of the authors and his advertising client agreed to devise a test involving three separate banner ad styles to measure any difference in response rates. The banner ads were identical in content, promoting a sports oriented video game. The ads were deployed on two popular web sites frequented by youths. The content of the ads has been removed to protect the anonymity of the client and advertising firm, however the size and shape of each banner ads is shown in Figure 1.

The effectiveness of the three banner ad styles was determined by comparing the relative click-through rates and interaction rates to the total number of impressions for each banner size. These terms are defined as follows:

- an impression is a measure of the number of times a particular ad is viewed;

- $\quad$ an interaction rate is the percentage of times an ad has been rolled over to launch or expand a panel;

- a click-through rate is the percentage of times an ad has been clicked to visit the site associated with the ad.
While click-through rates are the most common metric historically used to measure banner effectiveness, with the proliferation of rich media and expandable banners, interaction rates has quickly become the second most popular statistic [12].

The three different styles of banner ads, shown in Figure 1, were measured over an extended period of time, with over 10 million total impressions between the two sites and the three styles. The advertising agency used in this study charges the same rate for all three types of ads. Thus, a determination of the most effective style of ad could provide important implications for both clients who wish to optimize return on investment and web sites or advertising agencies who can use this information to guide decisions about costs and site optimization.

\section{<insert Figure 1 here>}

\section{RESULTS AND CONCLUSIONS}

As shown in Table 1, The 160 X 600 (wide skyscraper) style achieved the highest click through rate on both of the sites, more than doubling the rates of the other two styles. This style also outperformed the 728 X 90 (leaderboard) in the interaction rate metric for both sites. The 300 X 250 (medium rectangle) achieved the highest interaction rate in the second site.

\section{<insert Table 1 here $>$}

The relative effectiveness of the wide skyscraper banner is particularly interesting when compared to prior surveys and webinars conducted by search engine leaders, Google and Yahoo [14]. Yahoo's survey indicates that leaderboard positioning of banner ads is most effective, trailing only ads embedded within content as the most effective ad placement. In terms of size, the Google webinar states that the large rectangle ads tend to be the most effective, however, the author cautions that different styles may be more suitable for different types of content.

While this study attempts to isolate the effect of banner style, relative to content, other factors may play a role in these findings. The users of this site were primarily children and teenagers. Past data has shown that children's response to online advertisement may differ from responses from those of adult users. Young children (under 12 years of age), have been shown to have a significantly higher than average click-through rate, while teenagers (ages 
12-17) have a lower than average rate and the 18 to 20 year old group has an even lower rate [21]. Furthermore, this study does not attempt to separate size from orientation. A future study might include leaderboard or skyscraper banners of different sizes.

There is a wealth of data that web masters gather on a daily basis to optimize their sites, including the metrics of different styles of banner ads. However, few studies have been published in academic literature that strive to control the factors that may influence the rates. With the vast amounts of money being increasingly directed towards Internet advertising, it is important to examine the factors that influence the relative success of different types of advertisements. While this study is clearly just a starting point for examining these factors, it provides a foundation for other research on this and related subjects.

\section{REFERENCES}

1. Bruner, Rick E. "Best Practices for Optimizing Web Advertising Effectiveness." http://www.doubleclick.comlinsight/pdfs/dc bpwp 0605.pdf. May, 2006.

2. Business Week. "So Many Ads, So Few Clicks." November, 2007. http://www.businessweek.com/magazine/content /07_46/b4058053.htm

3. Business Wire. "DoubleClick's Research Demonstrates the Benefits of Online Video Advertising." March,2007.

4. Cho, Chang-Hoan. "Factors Influencing Clickers of Banner Ads on the WWW." CyberPsychology, Vol. 6, No. 2, 2003.

5. Cleland, Kim \& Carmichael, Matt. "Banners That Move Make a Big Impression." Advertising Age, Vol. 68, No. 2, 1997.

6. Dahlen, Micael. "Banner Advertisements Through a New Lens.” Journal of Advertising Research. July/August, 2001.

7. Danaher, Peter J. "Factors Affecting Online Advertising Recall; A Study of Students." Journal of Advertising Research. September, 2003.

8. Dreze, Xavier \& Hussherr, Francois-Xavier. "Internet Advertising: Is Anybody Watching?" Journal of Interactive Marketing. Vol. 17, No. 4. Autumn, 2003.

9. Forrester Research. "Barriers Between Traditional And Interactive Marketing Will Dissolve Within Five Years." October, 2007.

10. Fleischner, Michael. "7 Secrets for Increasing Internet Banner Ad Click-Through Rates." http://websitetips.comlartic!eslmarketing1banner adsctr/.
11. Hong, Chan-pyo. "A Snapshot Review of Research on Internet Advertising as Persuasive Communication." American Academy of Advertising Conference Proceedings, 2006.

12. Internet Advertising Bureau. "Rich Media Trends."

wwwjabuk.net/media/images/PointRolIRichMed iaTrendsforIAB 1175.PDF.

13. Jaffee, Larry. "Promo Annual Survey: Follow the Money." PROMO Magazine. May, 2007.

14. Kaiser, Shirley. "Banner Ads: The Best Performing Banner Ad Sizes, Formats, Locations, and Colors." http://websitetips.com/articles/marketing/bannera dsizes/.

15. Laudon, Kenneth \& Traver, Carol. E-commerce. Addison Wesley, 2001.

16. Li, Hairong. "What Makes the User Click on a Banner Ad: Two Field Experimental Studies of Banner Ad Size, Type, and Incentive.” Proceedings of the Conference of the American Academy of Advertising, 1998.

17. Li, Hairong \& Bukovac, Janice. "Cognitive Impact of Banner Ad Characteristics: An Experimental Study.” Journalism and Mass Communication Quarterly. Vol. 76, No. 2. Summer, 1999.

18. Lynch, Steve. "Digitrends.net: To Banner, or Not to Banner?" http://www.molineconsulting.comlReinventando/Pagines/eIAmbien te.htm.

19. Nielsen/NetRatings. http://www.nielsennetratings.com.

20. Pharr, Julie. "A Research Agenda for Brandbuilding on the Internet with Banner Advertising.” Services Marketing Quarterly. Vol. 26, No. 2, 2004.

21. Saunders, Christopher. "Study: Kids Click Banner Ads Most, Teens Least." The ClickZ Network.

http://www.clickz.com/showPage.html?page=43 7941. August, 2000.

22. Scott, Andrew. "The New Frontier." PROMO Magazine. April,2006.

23. Spethmann, Betsy. "Designs on Nascar." PROMO Magazine. May, 2007.

24. Wood, Rob. "Improving Your Click-through Rate." http://clixgaJoretutor.comlImprovingYour-Click-Through-Rate.shtml. 
TABLES AND FIGURES

Figure 1: Banner Styles

$$
728 \text { X } 90 \text { (Leaderboard) }
$$
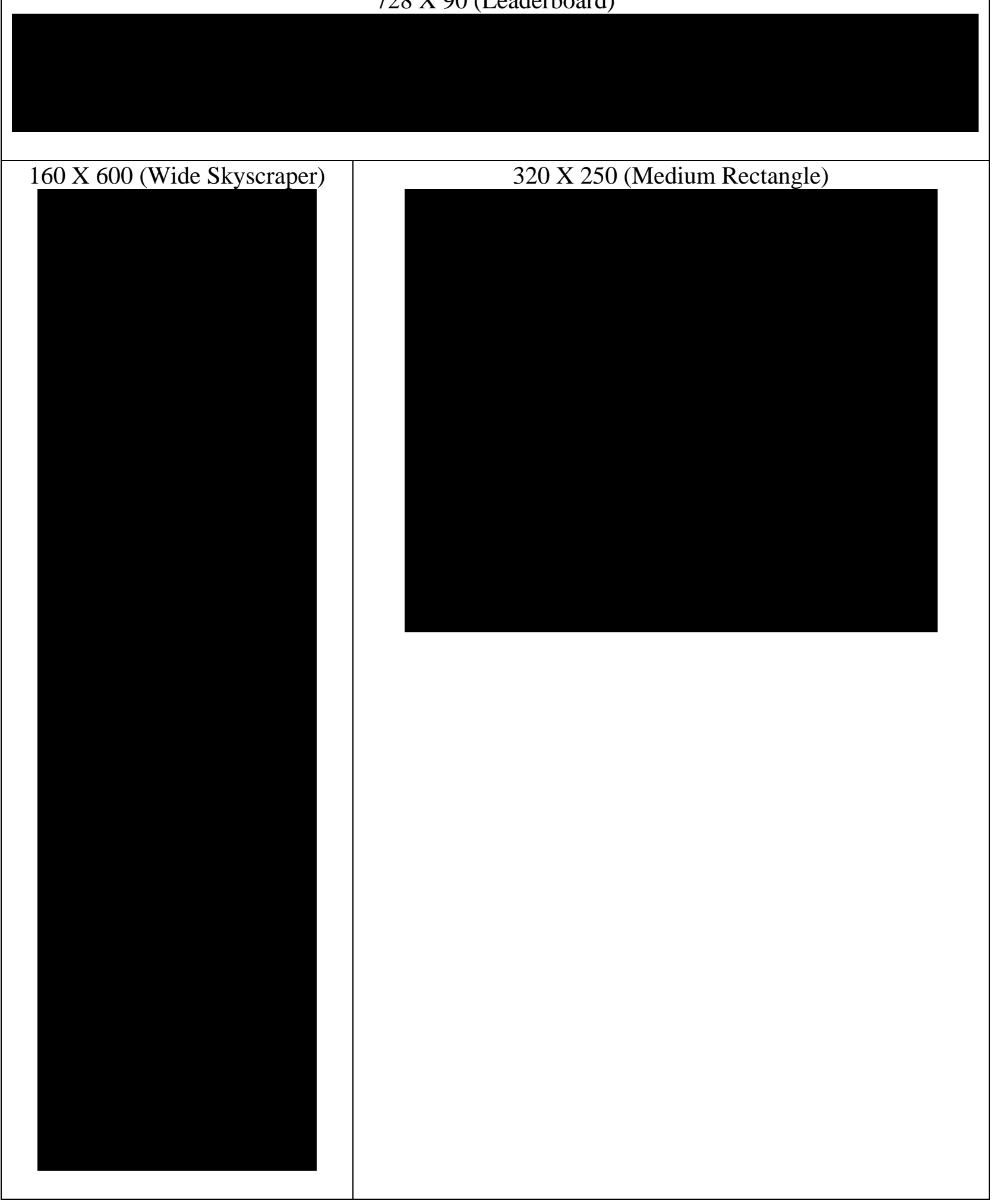
Table 1: Success Rates of Banner Ad Styles

\begin{tabular}{|c|c|c|c|c|c|c|}
\hline $\begin{array}{c}\text { Banner } \\
\text { Size }\end{array}$ & File Name & Impressions & Interactions & $\begin{array}{c}\text { Interaction } \\
\text { Rate }\end{array}$ & $\begin{array}{c}\text { Total } \\
\text { Clicks }\end{array}$ & $\begin{array}{l}\text { Total } \\
\text { CTR }\end{array}$ \\
\hline & & $8,560,696$ & 457,433 & 7.1608 & 30,688 & $0.358 \%$ \\
\hline \multirow{4}{*}{$160 \times 600$} & & $3,113,932$ & 193,077 & $10.901 \%$ & 22,698 & $0.729 \%$ \\
\hline & 160x600_GIF_Dflt & 780,569 & 0 & N/A & 5,229 & $0.670 \%$ \\
\hline & 160x600_Exp_DERIVE & $1,771,165$ & 190,834 & $10.774 \%$ & 7,973 & $0.450 \%$ \\
\hline & 160x600_FL_DERIVE & 562,198 & 2,243 & N/A & 9,496 & $1.689 \%$ \\
\hline \multirow[t]{3}{*}{$300 \times 250$} & & 810,735 & 1,593 & $0.202 \%$ & 840 & $0.104 \%$ \\
\hline & 300x250_GIF_Dflt & 23,580 & 0 & N/A & 65 & $0.276 \%$ \\
\hline & 300 $\times 250$ Polite_DERIVE & 787,155 & 1,593 & $0.202 \%$ & 775 & $0.098 \%$ \\
\hline \multirow[t]{4}{*}{$728 \times 90$} & & $3,437,481$ & 192,363 & $6.305 \%$ & 7,134 & $0.208 \%$ \\
\hline & 728x90_GIF_Dflt & 386,514 & 0 & N/A & 816 & $0.211 \%$ \\
\hline & 728x90_Exp_DERIVE & $3,050,967$ & 192,363 & $6.305 \%$ & 6,318 & $0.207 \%$ \\
\hline & & $2,101,407$ & 153,135 & $7.990 \%$ & 14,294 & $0.680 \%$ \\
\hline \multirow[t]{3}{*}{$160 \times 600$} & & 755,235 & 77,012 & $11.170 \%$ & 9,468 & $1.254 \%$ \\
\hline & 160×600_GIF_Dflt & 65,770 & 0 & N/A & 551 & $0.838 \%$ \\
\hline & 160×600_Exp_DERIVE & 689,465 & 77,012 & $11.170 \%$ & 8,917 & $1.293 \%$ \\
\hline \multirow[t]{3}{*}{$300 \times 250$} & & 126,686 & 17,589 & $15.382 \%$ & 760 & $0.600 \%$ \\
\hline & 300×250_GIF_Dflt & 12,340 & 0 & N/A & 56 & $0.454 \%$ \\
\hline & 300×250_Exp_DERIVE & 114,346 & 17,589 & $15.382 \%$ & 704 & $0.616 \%$ \\
\hline \multirow[t]{4}{*}{$728 \times 90$} & & $1,219,486$ & 58,534 & $5.260 \%$ & 4,066 & $0.333 \%$ \\
\hline & 728×90_GIF_Dflt & 106,615 & 0 & $\mathrm{~N} / \mathrm{A}$ & 234 & $0.219 \%$ \\
\hline & 728x90_Exp_DERIVE & $1,112,871$ & 58,534 & $5.260 \%$ & 3,832 & $0.344 \%$ \\
\hline & & $10,662,103$ & 610,568 & $7.351 \%$ & 44,982 & 0.4228 \\
\hline
\end{tabular}

